\title{
An Improved Artificial Fish Swarm Algorithm and Its Application
}

\author{
Mantao Wang ${ }^{1,}$, , Haitao Tang ${ }^{1, b}$, Jong $\mathrm{Mu}^{1, \mathrm{~b}}$ and Peng Wei $\mathrm{i}^{1, \mathrm{~d}}$ \\ ${ }^{1}$ College of Information and Engineering, Sichuan Agricultural University, 625014, China \\ awangmantao@126.com, b1076092539@qq.com, cscmjmj@yahoo.com.cn, d1063951438@qq.com
}

Keywords: Artificial Fish Swarm Algorithm (AFSA); Parameters dynamic mechanism; Iterative adaptive mechanism; Local traversal algorithm

\begin{abstract}
To overcome the standard AFSA's slow convergence speed and limited optimizing accuracy problem, an improved AFSA is presented in this paper. For this improved algorithm, parameters dynamic mechanism is introduced to improve the accuracy. Besides, iterative adaptive mechanism and local algorithm were introduced to overcome invalid calculation and convergence shocks problems. Comparison experiments show that the improved AFSA is better than the standard algorithm on the convergence rate and optimization accuracy. Moreover, SVM parameter optimization also shows that the improved AFSA has a better optimization performance metric, time performance metric and robustness metric than traditional method.
\end{abstract}

\section{Introduction}

Artificial Fish Swarm Algorithm (AFSA) is a Chinese scholars Ding Xiaolei [1] put forward a new kind of intelligent optimization Algorithm for the first time in his doctoral thesis. AFSA simulates fish for food, according to the group, collision, random walk basic behavior, using bottom-up ideas for optimization, belongs to the artificial intelligence algorithm based on behavior. At present, based on the research of artificial fish algorithm has been penetrated into multiple applications, and received very good practical application, the current is one of research hotspots in the field of swarm intelligence algorithm [2]. Artificial fish algorithm has faster convergence speed, and strong robustness, the initial parameters dependence is small, it can quickly search the feasible solution range of advantages and is very suitable for solving optimization problem of accuracy which is not high [3]. However, the artificial fish algorithm is slow convergence in the late time at the same time, there are also two major defects about limited precision.

Aiming at the two defects of artificial fish algorithm, many domestic and international scholars carried on a great deal of empirical research to this and found some practical and feasible optimization schemes. For example: Song Xiaoxiao was proposed based on polar coordinates coding about initialization algorithm in her literature [4]; Guang-zhou Chen, introduced the immune algorithm in the demise of operator and compared and abandon the optimal individual to a certain extent to improve the convergence speed of artificial fish algorithm in his literature [5];Dong-dong Wang's team proposed the optimization of the segment and accelerate the convergence speed of the algorithm to a certain extent in theri literatures [6];Luo Dexiang is put forward to particle swarm and artificial fish hybrid optimization algorithm to a certain extent to improve the accuracy of the algorithm in his literature [7]; Li liang used to construct two taboo optimization operator the way to the repeated search the solution space and ensure the algorithm which can overcome the local optimal problem, to a certain extent, to improve the efficiency of the algorithm in his literature [8].At present, foreign scholars on the research of the artificial fish algorithm is far less Chinese scholars, but there are still gains in some aspects. for example, paper [9] put forward a kind of artificial fish algorithm, based on filters, Ana Maria team will filter algorithm based on population-based filter algorithm combined with artificial fish algorithm, and successfully used to solve the nonconvex constraint optimization problems, a preliminary numerical experiments show that the algorithm than the original artificial fish algorithm which is more competitive on the convergence rate. 
Above of artificial fish algorithm to optimize a certain extent, it solves some disadvantages of the standard algorithm, especially the most improvement for the parameters of the artificial fish are studied, but the current study of artificial fish algorithm "iterations" this parameter is not covered, and the experiment and engineering practice shows the number of iterations have a great influence on the timing of the artificial fish algorithm. To solve above problems, this paper introduced the iterative adaptive termination mechanism and parameter adaptive mechanism, and combined with local time calendar calculation method for standard artificial fish algorithm in the optimization, the results show that the algorithm has large improvement in performance.

\section{The Basic Principle of Artificial Fish Algorithm}

The visual system of the fish is very advanced, it can quickly perceive things a lot of space. AFSA takes advantage of the model to simulate the basic movement of artificial fish in Fig. 1.

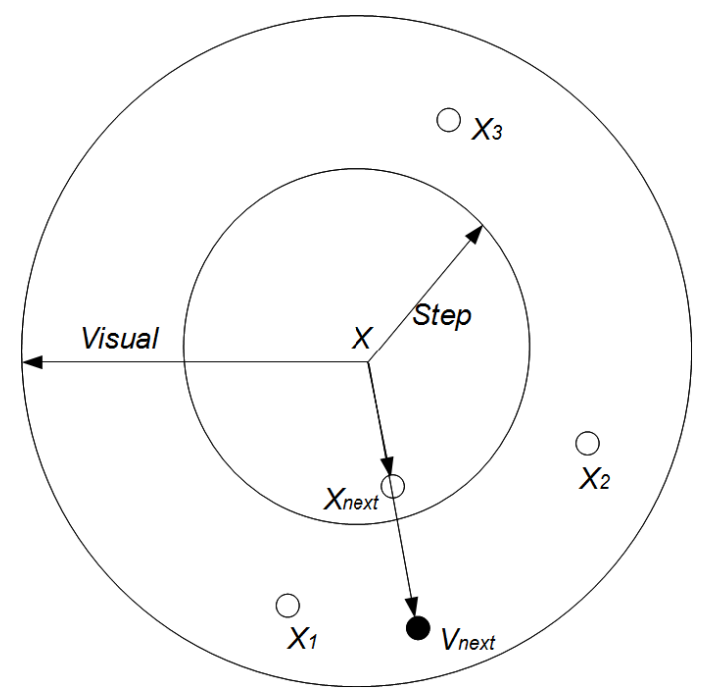

Figure 1. Basic model of AFSA

In Fig. 1, let to a current position of artificial fish for $X\left(X=\left(x_{1}, x_{2}, x_{3} \cdots x_{n}\right)\right)$, its visual length is Visual, every time a maximum distance of can act as Step, $X_{i}(i=1,2,3 \cdots)$ is on behalf of the other artificial fish. Artificial fish in the phase of the visual field of random search of $V_{\text {next }}\left(V_{n \text { next }}=\left(x_{1}^{v}, x_{2}^{v}, x_{3}^{v} \cdots x_{n}^{v}\right)\right)$ said some point to the location of the artificial fish viewpoint, if things concentrations of $V_{\text {next }}$ (function value) is greater than the $X$ position of the food concentration, so artificial fish next to the vector of the $V_{\text {next }}$ direction, moving the location of the record for $X_{\text {next }}$. The diagram, the calculation of $V_{\text {next }}$ and $X_{\text {next }}$ as formula (1), (2), among them, $r$ is $[-1,1]$ range of random Numbers.

$$
\begin{aligned}
& x_{i}^{v}=x_{i}+\text { Visual } \cdot r, i=1,2,3 \cdots n \\
& X_{n e x t}=\frac{X_{v}-X}{\left\|X_{v}-X\right\|} \cdot \text { Step } \cdot r+X
\end{aligned}
$$

Artificial fish algorithm mainly includes the fish foraging, bunching, collision and random about four kinds of behavior. Combination of the four behavior and cooperation to realize the artificial fish in closed SuoYu ( function domain optimization). Using artificial fish algorithm optimization flow chart is shown in Fig. 2. 


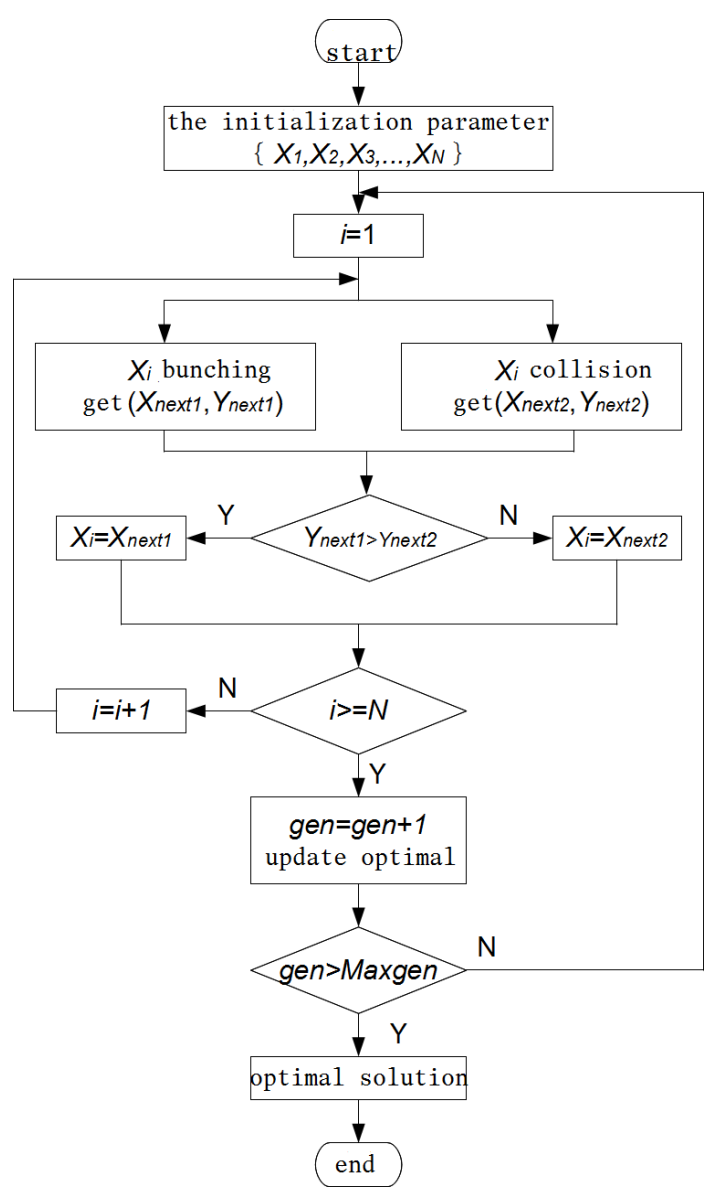

Figure 2. Flow Diagram of AFSA

\section{Improved Artificial Fish Algorithm Is Introduced and the Implementation}

In the standard artificial fish algorithm, Step, Visual two parameters are fixed, bigger Step, Visual can guarantee fast convergence at the early stage of the algorithm parameter values, but large parameters will effect the precision of algorithm optimization in late, and even lead to the search results only local optimal value. For balancing algorithm's convergence speed and precision of the dynamic parameters are introduced regulate factor $\lambda(0<\lambda<1)$, let Step, Visual parameters to dynamic changes in the process of iteration (formula (3), (4)) in the improved artificial fish algorithm. Early use of larger parameter values ensure early rapid convergence algorithm; Late parameter decreases and ensure the search accuracy. In the movement of artificial fish, because of the standard artificial fish algorithm without pass the optimal value "real-time" information foraging behavior, cluster and fundation of artificial fish, namely the global optimal value failed to timely in the optimization of artificial fish every exploration effect. To overcome the disadvantages, the artificial fish cluster and train the mobile reference factor in expanded from the original food concentration centre for food concentration center combined with the global optimal position in foraging behavior. for example, it was revised formula (6), packed and moved to correct the same crash behavior in the mobile behavior of foraging behavior by the standard algorithm in the formula (5) .

$$
\begin{aligned}
& \text { Visual=Visual } \cdot(1-\lambda) \\
& \text { Step }=\text { Step } \cdot(1-\lambda)
\end{aligned}
$$




$$
\begin{aligned}
& X_{\text {next }}=\frac{X_{v}-X}{\left\|X_{v}-X\right\|} \cdot \text { Step } \cdot r+X \\
& X_{\text {next }}=\frac{X_{\text {best }}+X_{v}-2 \cdot X}{\left\|X_{\text {best }}+X_{v}-2 \cdot X\right\|} \cdot \text { Step } \cdot r+X
\end{aligned}
$$

In formula (6), $X$ represents the global optimal position of food concentration. Using artificial fish algorithm search out the optimal value which is the optimal value of objective function theory for continuous function optimization, so it is possible to get as high as a limited time precision that it is the key of the artificial fish algorithm optimization algorithm. Experimental data show that artificial fish algorithm late iteration of the effect on accuracy function finally, belong to "invalid iterative calculation in literature [9].Late to eliminate the waste and improve the artificial fish algorithm of rapidity and precision of the permitted error introduced precision $\mathrm{K}$ and iterative adaptive termination number $\mathrm{Z}$, and connecting with the grid search method, make the artificial fish after the operation accuracy of convergence to the range of allowable error timely termination of iteration, saves the operation time. As a result of the existence of random behavior in artificial fish algorithm for computing the global optimal solution of the late, after the expiration of the iteration, a local grid traversal can overcome much of random behavior influence on final precision, improve the computing accuracy. Improved artificial fish algorithm process as shown in Fig. 3:

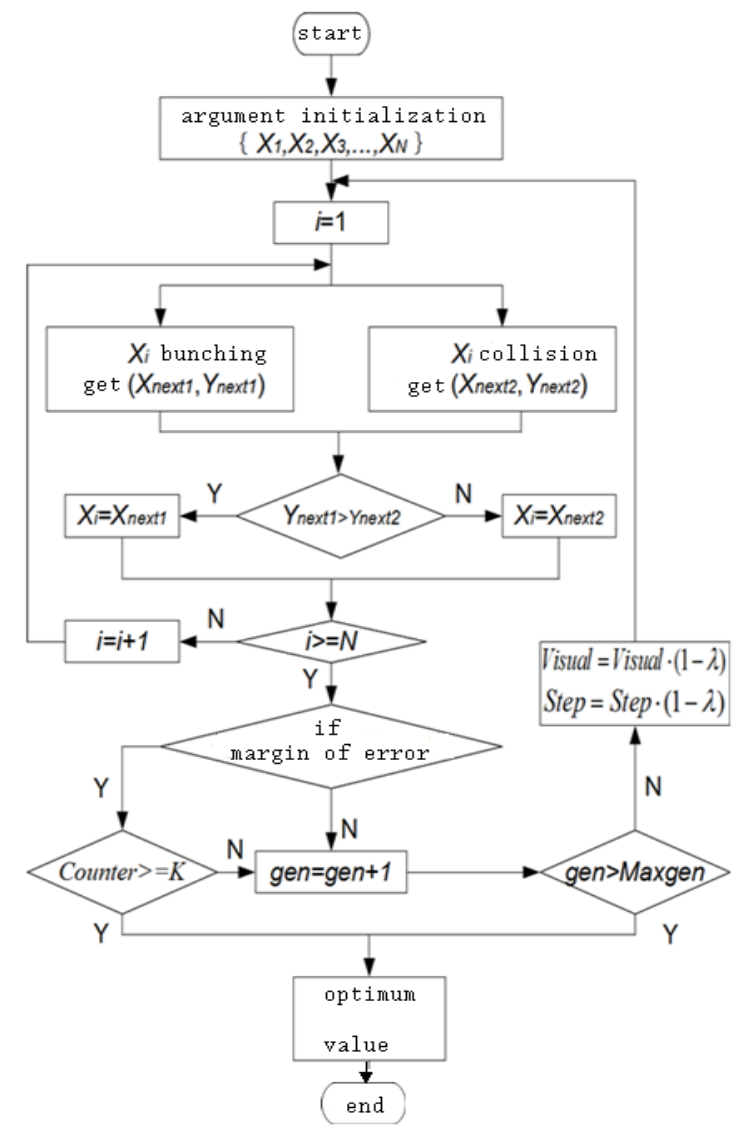

Figure 3. Flow Diagram of Improved AFSA 
The realization method of improved artificial fish algorithm is as follows

The name of Algorithm: Improved Artificial Fish Swarm Algorithm, IAFSA

1. Input: Let objective function is $f(X)$, artificial fish for $N$, the largest number of iterations Maxgen, maximum test times try_number, Visual is visual perception distance , $\delta$ is crowded degree, moving step length is Step, parameter dynamic adjustment factor $\lambda, \varepsilon$ is permitted error precision, iterative adaptive termination number is $K$.

2. Let $N$ artificial fish evenly into the objective function $f(X)$ the independent variable within the scope of article $N$ of the artificial fish cluster and in turn rear-ended behavior after comparison to determine the final location of $X_{\text {next }}^{i}(i=1,2,3 \cdots, N)$, initializes the global optimal value $Y_{b e s t}^{1}$ position with $X_{b e s t}^{1}$, namely initializes the bulletin board.

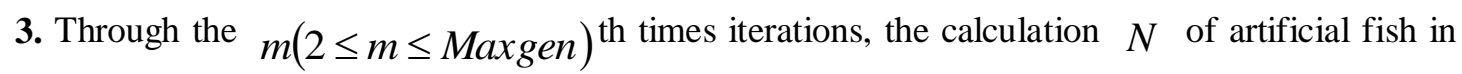
turn of cluster compared with collision behavior after moving the final location of $X_{\text {next }}^{i}(i=1,2,3 \cdots, N)$ and concentration $Y_{\text {next }}^{i}(i=1,2,3 \cdots, N)$ things, calculate I update the global optimal value $Y_{\text {best }}^{m}$ and position of $X_{\text {best }}^{m}$.If $m<$ Maxgen jump step 4, $m=$ Maxgen will jump step 5 .

4. According to the formula (7) whether the calculation results of the first $m$ iterations within the permitted error precision $\varepsilon$.If meet the precision of allowable error counter plus one, if counter to iterative adaptive termination number $K$, skiping to step 5, otherwise, skiping to step 3 to continue iterative calculation;If permitted error does not meet the precision, counter reset skip to step 3 to continue the iterative calculation.

$$
\left\|Y_{\text {best }}^{m}-Y_{\text {best }}^{m-1}\right\| \leq \varepsilon^{2}(m=2,3, \cdots, \text { Maxgen })
$$

5. Thought the grid search, pseudo code is as follows. The independent variable $X$ is the dimension of $n \times 1\left(n \geq 1, n \in N^{*}\right)$ matrix, the independent variable $X$ lets $i(i=1,2, \cdots n)$ th for $X^{i}$.

$$
\begin{aligned}
& \text { for }\left(i 1=X^{1}-\text { Visual } ; i 1 \leq X^{1}+\text { Visual } ; i 1=i 1+\text { Step } / 10\right) \\
& \text { for }\left(i 2=X^{2}-\text { Visual } ; i 2 \leq X^{2}+\text { Visual } ; i 2=i 2+\text { Step } / 10\right) \\
& \vdots \\
& \text { for }\left(i i=X^{i}-\text { Visual } ; i i \leq X^{i}+\text { Visual } ; i i=i i+\text { Step } / 10\right) \\
& \{ \\
& \text { if }\left(f(X)>Y_{\text {best }}\right) \\
& Y_{b e s t}=f(X) ; \\
& \{
\end{aligned}
$$

6. Output: $Y_{\text {best }}$.

\section{The Experimental Results and Analysis}

Improved Artificial Fish Algorithm Simulation Contrast Test. To explore the improved artificial fish algorithm relative standard artificial fish algorithm on the algorithm performance is improved and designed a set of contains a maximum of 10 different types of function optimization simulation experiments, and contrast experiment related parameters such as Table 1, the experimental results are recorded in Table 2.In this section, the simulation using the computer platform for Windows 7 (64) system, the processor as the core i7, memory is 8G. experiments using software simulation platform for Matlab2013a. 
Contrast test evaluation indexes corresponding algorithm used in current academia: that are widely used in the optimization performance index, time performance and robustness index [13], the relevant mathematical definition respectively, such as formula (8), (9), (10).

$$
\begin{aligned}
& D_{m}=\sqrt{\frac{\sum_{i=1}^{n}\left(C_{b, i}-C^{*}\right)^{2}}{n}} \\
& E_{t}=\frac{\sum_{i=1}^{n} T_{i}}{n} \\
& E_{f}=\sqrt{\frac{\sum_{i=1}^{n}\left(C_{i}-\bar{C}\right)^{2}}{n}}
\end{aligned}
$$

Formulas (8), $C^{*}$ is the theory of the optimal solution of the problem, the theory of optimal solution is unknown or cannot work out can be known optimal solution, repeat operation $i$ times $C_{b, i}$ representative algorithm after the actual optimal value of the optimal value standard deviation $D_{m}$ as evaluation index of algorithm precision, contrast experiment in each group headed by five rounds of testing. Formula (9), $T_{i}$ representative algorithm first time $i$ time consuming of the optimal value, the smaller $E_{t}$ means that the faster the algorithm convergence speed, namely the timeliness, the better. Formula (10), $C_{i}$ represents the value of the algorithm is the $i$ th times which you run. $\bar{C}$ said algorithm running $n$ times average. $E_{f}$ the smaller the robustness of the algorithm (reliability).

Table 1 Parameters of Simulate Testing

\begin{tabular}{cccccccccc}
\hline Parameter Algorithm & $N$ & Visual & Step & try_number & Maxgen & $\delta$ & $K$ & $\lambda$ & $\varepsilon$ \\
\hline AFSA & 50 & 1 & 1 & 30 & 50 & 0.618 & $/$ & $/$ & $/$ \\
IAFSA & 50 & $1 *(1-\lambda)$ & $1 *(1-\lambda)$ & 30 & 50 & 0.618 & 10 & 0.05 & 0.01 \\
\hline
\end{tabular}

Algorithm performance simulation contrast from data analysis in the results (Table 2) : because of the improved artificial fish algorithm implemented parameters dynamically setting and joined the local grid search, compared with the standard artificial fish algorithm the optimization result is more close to theoretical values, most optimal performance parameters and robustness are smaller; In terms of time performance, due to the improved artificial fish algorithm joined the "iterative adaptive mechanism", its optimal spent significantly less than the standard algorithm, timing algorithm is improved.

Improved Artificial Fish Algorithm in the Application of Support Vector Machine (SVM) Parameters Optimization. In order to further explore the improved artificial fish algorithm under the condition of complex function to explore the ability of maximum value, this paper the improved artificial fish algorithm using the support vector machine (SVM) parameters optimization experiment. 
Table 2 Comparison of AFSA and IAFSA Performance

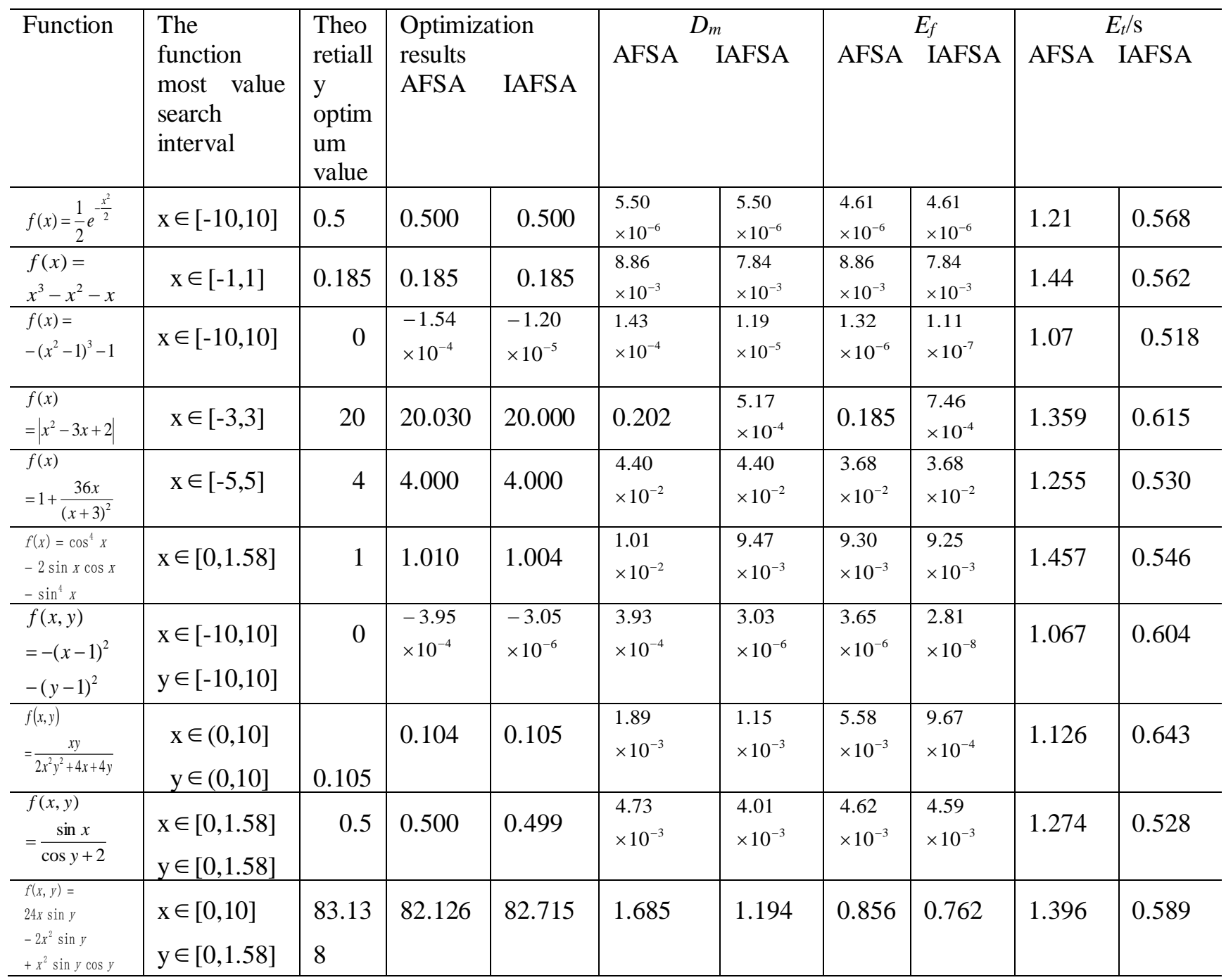

$\left(D_{m}:\right.$ Optimal performance, $\quad E_{f}:$ Robust Performance,$\quad E_{t} / \mathrm{s}$ : Time performances )

SVM (Support Vector Machine, SVM) is a kind of based on statistics of VC dimension theory and structure risk minimum principle of supervised learning algorithm [10].The SVM is widely used in data classification task, before using the SVM parameters optimization training must be applied on them. At present, how to choose the parameters of the SVM effectively has not theoretical guidance, the commonly used method with grid method, fifty percent $[11,12]$ cross validation method. This year, with the development of the group of intelligent algorithm, many scholars have already put such as particle swarm optimization (PSO), artificial fish algorithm such as swarm intelligence algorithm used in the parameter optimization of SVM [13].

Support vector machine (SVM) parameters optimization experiment, compared the grid method, cross validation method is fifty percent, the standard artificial fish optimization method and the improved artificial fish optimization method in this paper. In order to guarantee the effectiveness of experiment contrast, in the simulation experiments, the SVM function using Libsvm standard RBF SVM function provided by the website, the contrast experiment on standard RBF - gamma, C parameters of the SVM function optimization. Other relevant parameter Settings as shown in Table 3 , in addition to the grid method, fifty percent cross validation method, AFSA and IAFSA optimization search range is set to gamma $\in[0.01,10], C \in[0.01,10]$.

Support vector machine (SVM) parameters optimization experiments were set up eight different 
sets of data, data details as shown in Table 4.Fifty percent due to the grid method, cross validation method, IAFSA AFSA and this article research on the principle of algorithm has very big difference, cannot use unified algorithm evaluation index, based on support vector machine (SVM) parameters optimization experiment selection algorithm running time as evaluation index, timeliness of choosing the evaluation accuracy of the SVM and discriminant accuracy as the algorithm to optimize performance.

In contrast experiments, each group of data of five rounds to repeat the experiment. Among them, the time consuming of parameter optimization in support vector machine (SVM) (Table 5) in the experiment data of five round experiment of average; The accuracy of parameter optimization in support vector machine (SVM) comparative trials (Table 6), the SVM discriminant accuracy and evaluation accuracy take five times the maximum of experiment.

Table 3 Parameters of SVM optimization test

\begin{tabular}{lllrrrrrrc}
\hline $\begin{array}{l}\text { Parameter } \\
\text { Algorithm }\end{array}$ & $N$ & Visual & Step & try_number & Maxgen & $\delta$ & $K$ & $\lambda$ & $\varepsilon$ \\
\hline AFSA & 30 & 1 & 1 & 30 & 50 & 0.618 & $/$ & $/$ & $/$ \\
IAFSA & 30 & $1 *(1-\lambda)$ & $1 *(1-\lambda)$ & 30 & 50 & 0.618 & 10 & 0.05 & 0.01 \\
Grid Method & $/$ & $/$ & 0.01 & $/$ & $/$ & $/$ & $/$ & $/$ & $/$ \\
Fifty Percent & $/$ & $/$ & 0.01 & $/$ & $/$ & $/$ & $/$ & $/$ & $/$ \\
$\begin{array}{l}\text { Cross Validation } \\
\text { Method }\end{array}$ & & & & & & & & & \\
\hline
\end{tabular}

Table 4 Data of SVM optimization test

\begin{tabular}{lccccc}
\hline $\begin{array}{l}\text { Data } \\
\text { Characteristics }\end{array}$ & $\begin{array}{c}\text { Data } \\
\text { Dimension }\end{array}$ & $\begin{array}{c}\text { Data } \\
\text { Category }\end{array}$ & $\begin{array}{c}\text { Training } \\
\text { Scale }\end{array}$ & $\begin{array}{c}\text { Scale } \\
\text { Evaluation }\end{array}$ & $\begin{array}{c}\text { Measurement } \\
\text { Scope }\end{array}$ \\
\hline wine & 13 & 3 & 96 & 24 & 58 \\
Iris & 4 & 3 & 96 & 24 & 30 \\
Beast Tissue & 9 & 6 & 71 & 18 & 17 \\
Libras ovement & 90 & 15 & 240 & 60 & 60 \\
Statlog & 18 & 4 & 752 & 150 & 94 \\
east & 8 & 10 & 959 & 241 & 284 \\
Segment & 19 & 7 & 1600 & 400 & 310 \\
Optical & 64 & 10 & 3060 & 763 & 1797 \\
\hline
\end{tabular}

Table 5 Time-consuming of SVM optimization test

\begin{tabular}{lcccc}
\hline Data Contrast & \multicolumn{4}{c}{ The SVM } \\
& GM & CVM & AFSA & IAFSA \\
\hline wine & 7.562 & 32.432 & 58.613 & 12.904 \\
Iris & 6.442 & 22.274 & 45.312 & 10.012 \\
Beast Tissue & 6.633 & 32.475 & 67.503 & 9.327 \\
Libras Movement & 159.933 & 342.063 & 603.722 & 274.894 \\
Statlog & 115.208 & 876.466 & 1163.336 & 174.042 \\
Yeast & 224.376 & 1118.140 & 1630.144 & 198.150 \\
Segment & 376.791 & 1360.237 & 2108.530 & 244.510 \\
Optical & 8043.050 & 35267.739 & 40741.306 & 6398.772 \\
\hline
\end{tabular}


Table 6 Accuracy of SVM optimization test

\begin{tabular}{lcccccccc}
\hline $\begin{array}{l}\text { Data } \\
\text { Contrast }\end{array}$ & \multicolumn{3}{c}{ SVM Assessment The Accuracy } & \multicolumn{5}{c}{ SVM Judgement Right Rate } \\
& GM & CVM & AFSA & IAFSA & GM & CVM & AFSA & IAFSA \\
\hline wine & $100 \%$ & $99.17 \%$ & $100 \%$ & $100 \%$ & $96.55 \%$ & $98.28 \%$ & $100 \%$ & $100 \%$ \\
Iris & $100 \%$ & $95.83 \%$ & $100 \%$ & $100 \%$ & $86.67 \%$ & $86.67 \%$ & $93.33 \%$ & $96.67 \%$ \\
$\begin{array}{l}\text { Beast } \\
\text { Tissue }\end{array}$ & $83.33 \%$ & $73.03 \%$ & $83.33 \%$ & $66.67 \%$ & $58.82 \%$ & $52.94 \%$ & $64.71 \%$ & $64.71 \%$ \\
$\begin{array}{l}\text { Libras } \\
\text { Movement }\end{array}$ & $76.67 \%$ & $76.67 \%$ & $76.67 \%$ & $76.67 \%$ & $65.00 \%$ & $66.67 \%$ & $60.00 \%$ & $66.67 \%$ \\
Statlog & $86.00 \%$ & $84.04 \%$ & $84.00 \%$ & $82.67 \%$ & $97.87 \%$ & $94.68 \%$ & $97.87 \%$ & $99.36 \%$ \\
Yeast & $64.32 \%$ & $60.50 \%$ & $64.73 \%$ & $63.07 \%$ & $58.45 \%$ & $59.15 \%$ & $58.80 \%$ & $60.21 \%$ \\
Segment & $95.50 \%$ & $96.90 \%$ & $95.75 \%$ & $94.25 \%$ & $97.42 \%$ & $96.45 \%$ & $96.45 \%$ & $97.48 \%$ \\
Optical & $99.21 \%$ & $99.29 \%$ & $99.58 \%$ & $99.58 \%$ & $98.50 \%$ & $98.33 \%$ & $98.33 \%$ & $98.50 \%$ \\
\hline
\end{tabular}

By analyzing the experimental data shows that the grid method in the optimization of small sample data takes the shortest, but the optimal discriminant accuracy assessment of the accuracy and the gap between the larger, the parameters obtained from the optimization of discriminant accuracy is low, and in the case of large data time-consuming than improved artificial fish algorithm; Fifty percent relative to the grid method of cross validation algorithm, the closer to the discriminant accuracy assessment accuracy, but the algorithm is time-consuming have multiplied several relative grid method, takes in four types of methods is bigger, does not apply to big data, and ultimately discriminant accuracy is generally less than AFSA and IAFSA;AFSA, compared with fifty percent grid method and the accuracy of cross validation algorithm with discriminant accuracy is generally higher, and the optimization of higher precision, but AFSA takes is generally highest in four ways, the same does not apply to large data environment; IAFSA spent close to grid method, and takes the lowest in the big data environment, is suitable for large data in four ways a kind of environment. In terms of optimization accuracy, IAFSA discriminant accuracy is higher than other common evaluation accuracy and 3 classes. Overall, IAFSA is a kind of good ageing performance and optimize the performance of the algorithm, suitable for parameter optimization of support vector machine (SVM).

\section{Summary}

In this paper, a new kind of swarm intelligence algorithm, artificial fish algorithm in the study of optimization. By introducing the iterative adaptive mechanism to effectively control the overall number of iterations, artificial fish algorithm overcomes the standard artificial fish algorithm late due to invalid iteration calculation of waste, improve the efficiency of the algorithm; In the artificial fish algorithm of cluster, collision with the introduction of the parameters of the dynamic random behavior mechanism to improve the algorithm convergence speed; Iteration after expiration of the algorithm to optimize accuracy requirement, and combined with the local grid through calendar calculation method, the greatest degree to overcome the local optimal value. Simulation experiment proves that the improved artificial fish algorithm in optimization of sex, timeliness and robustness are better than the standard artificial fish algorithm, in the parameter optimization of support vector machine (SVM) experiments, the modified artificial fish algorithm also show the time consuming is small, the advantages of high precision. However, in this paper, the optimization of artificial fish algorithm is still in the initial exploration stage, there are still many places need to explore with artificial fish algorithm optimization, such as: parameters should be how to dynamically adjust the crowded degree, the number of the artificial fish should be how to adapt to the change of the number of iterations, etc., such problems are the direction of the next step of work and motivation. 


\section{Acknowledgement}

Construction of big data hierarchical storage model for agricultural Internet of things, 15ZB0017

\section{References}

[1] X.L. Li: A new type of intelligent optimization method-AFSA. (Doctoral Dissertation of Zhejiang University, China 2003), p.24.

[2] K. Zou, T. Liu, W.W. Bao: Review of Research in AFSA. Vol.2 (2012) No.2, p.1674.

[3] P. Yong: An improved artificial fish swarm algorithm for optimal operation of cascade reservoirs. Vol. 4 (2011) No.3, p.740.

[4] X.X. Song, D.H. Sun, J. Xie: The improved AFSA based on polar cod, Vol.10 (2010) No.3, p.2248.

[5] G.Z. Chen, J.Q. Wang, C.J. Li: An improved AFSA and its application, Vol.7 (2009) No.12, p.105.

[6] D.D. Wang, Z. Li, L. Liang Li: Based on the improved AFSA for solving systems of nonlinear multivariable equations (China, November 8-10, 2009). Vol. 4, p.163.

[7] D.X. Luo, Y.Q. Zhou, H.J. Huang: Hybrid PSO and AFSA, Vol.6 (2009) No.10, p.1257.

[8] L. Li, S.C. Chi, G. Lin: Taboo fish algorithm and its application in slope stability analysis, Vol.3 (2006) No.23, p.6.

[9] A.M. Rocha, M. Fernanda: A filter-based artificial fish swarm algorithm for constrained global optimization, Vol.6 (2014) No.10, p.239.

[10]C. Kan: New clustering algorithms for the support vector machine based hierarchical classification, vol.31 (2010) No.5, p.1285.

[11]D. Zhao: SVM Kernel function and the selection algorithm, Vol.9 (2014) No.2 p.226.

[12] S.L. Zhou, J.S. Liao: Nuclear parameter selection method of RBF-SVM and its application in fault diagnosis, Vol.28 (2014) No.3, p.240.

[13] M.J. Tan, H.M. Zhang, Y.Q. Lv: Swarm intelligence algorithm, Vol.12 (2013) No.8 p.142. 